\title{
Residual and Past Entropy for Concomitants of Ordered Random Variables of Morgenstern Family
}

\author{
M. M. Mohie EL-Din, ${ }^{1}$ M. M. Amein, ${ }^{1,2}$ Nahed S. A. Ali, ${ }^{3}$ and M. S. Mohamed ${ }^{3}$ \\ ${ }^{1}$ Department of Mathematics, Faculty of Science, Al-Azhar University, Cairo 11884, Egypt \\ ${ }^{2}$ Department of Mathematics and Statistics, Faculty of Science, Taif University, Hawia 888, Saudi Arabia \\ ${ }^{3}$ Department of Mathematics, Faculty of Education, Ain Shams University, Cairo 11341, Egypt
}

Correspondence should be addressed to M. S. Mohamed; jin_tiger123@yahoo.com

Received 25 June 2015; Accepted 7 September 2015

Academic Editor: Chunsheng Ma

Copyright (C) 2015 M. M. Mohie EL-Din et al. This is an open access article distributed under the Creative Commons Attribution License, which permits unrestricted use, distribution, and reproduction in any medium, provided the original work is properly cited.

For a system, which is observed at time $t$, the residual and past entropies measure the uncertainty about the remaining and the past life of the distribution, respectively. In this paper, we have presented the residual and past entropy of Morgenstern family based on the concomitants of the different types of generalized order statistics (gos) and give the linear transformation of such model. Characterization results for these dynamic entropies for concomitants of ordered random variables have been considered.

\section{Introduction}

The Morgenstern family discussed in [1] provides a flexible family that can be used in such contexts, which is specified by the distribution function (df) and the probability density function (pdf), respectively, as follows:

$$
\begin{aligned}
& F_{X, Y}(x, y) \\
& \quad=F_{X}(x) F_{Y}(y)\left[1+\alpha\left(1-F_{X}(x)\right)\left(1-F_{Y}(y)\right)\right], \\
& f_{X, Y}(x, y) \\
& \quad=f_{X}(x) f_{Y}(y)\left[1+\alpha\left(2 F_{X}(x)-1\right)\left(2 F_{Y}(y)-1\right)\right],
\end{aligned}
$$

where $-1 \leq \alpha \leq 1$, and $f_{X}(x), f_{Y}(y)$ and $F_{Y}(y), F_{Y}(y)$ are the marginal pdf and $\mathrm{df}$ of $X$ and $Y$, respectively. The parameter $\alpha$ is known as the dependence parameter of the random variables $X$ and $Y$. If $\alpha$ is zero, then $X$ and $Y$ are independent.

The general theory of concomitants of order statistics has originally been studied by David et al. [2]. Let $\left(X_{i}, Y_{i}\right), i=$ $1,2, \ldots, n$, be $n$ pairs of independent random variables from some bivariate population with $\mathrm{df} F(x, y)$. Let $X_{(r: n)}$ be the $r$ th order statistics; then the $Y$ value associated with $X_{(r: n)}$ is called the concomitant of the $r$ th order statistics and is denoted by $Y_{[r: n]}$. The pdf of $Y_{[r: n]}$ is given by

$$
g_{[r: n]}(y)=g_{Y_{[r: n]}}(y)=\int_{-\infty}^{\infty} f_{Y \mid X}(y \mid x) f_{(r: n)}(x) d x
$$

where $f_{(r: n)}(x)$ is the pdf of $X_{(r: n)}$.

The concept of gos was introduced by Kamps [3] and we refer to it as case-I of gos; Kamps and Cramer [4] have introduced a second model of gos which we refer to as caseII of gos. The concept of lower gos was given by Pawlas and Szynal [5], and later Burkschat et al. [6] introduced it as dual generalized order statistics (dgos) to enable a common approach to descendingly ordered random variables like reversed order statistics and lower records models.

For the Morgenstern family with pdf given by (2), the pdf of the concomitant of case-I of gos $Y_{[r, n, m, k]}, 1 \leq r \leq n$, is given by Beg and Ahsanullah [7] as follows:

$$
\begin{aligned}
& g_{[r, n, m, k]}(y) \\
& \quad=f_{Y}(y)\left[1+\alpha C^{*}(r, n, m, k)\left(2 F_{Y}(y)-1\right)\right],
\end{aligned}
$$


and the pdf of the concomitant of case-I of dgos $Y_{d[r, n, m, k]}$, $1 \leq r \leq n$, is given by Nayabuddin [8] as follows:

$$
\begin{aligned}
& g_{d[r, n, m, k]}(y) \\
& \quad=f_{Y}(y)\left[1-\alpha C^{*}(r, n, m, k)\left(2 F_{Y}(y)-1\right)\right],
\end{aligned}
$$

where $C^{*}(r, n, m, k)=1-2 \prod_{j=1}^{r} \gamma_{j} / \prod_{i=1}^{r}\left(\gamma_{i}+1\right), \gamma_{r}=k+(n-$ $r)(m+1), n \in \mathbb{N}, k \geq 1$, and $m_{1}=\cdots=m_{n-1}=m \in \mathbb{R}$. The pdf of the concomitant of case-II of gos $Y_{[r, n, \widetilde{m}, k]}, 1 \leq r \leq n$, is given by Mohie El-Din et al. [9] as follows:

$$
g_{[r, n, \widetilde{m}, k]}(y)=f_{Y}(y)\left[1+\alpha D_{[r, n, \widetilde{m}, k]}\left(2 F_{Y}(y)-1\right)\right],
$$

and the pdf of the concomitant of case-II of dgos $Y_{d[r, n, \widetilde{m}, k]}$, $1 \leq r \leq n$, is given by

$$
g_{d[r, n, \widetilde{m}, k]}(y)=f_{Y}(y)\left[1-\alpha D_{[r, n, \widetilde{m}, k]}\left(2 F_{Y}(y)-1\right)\right],
$$

where $D_{[r, n, \widetilde{m}, k]}=\left[1-2 c_{r-1} \sum_{i=1}^{r}\left(a_{i}(r) /\left(\gamma_{i}+1\right)\right)\right], a_{i}(r)=$ $\prod_{j=1, i \neq j}^{r}\left(1 /\left(\gamma_{j}-\gamma_{i}\right)\right), \gamma_{j} \neq \gamma_{i}, 1 \leq i \leq r \leq n, c_{r-1}=\prod_{j=1}^{r} \gamma_{j}$, $1 \leq r \leq n$.

Let $X$ be an absolutely continuous nonnegative random variable having $\mathrm{df} F(t)=P(X \leq t)$ and the survival function $\bar{F}(t)=P(X \geq t)$. Suppose $X$ denotes the lifetime of a component/system or of a living organism and $f(t)=F^{\prime}(t)$ denotes the lifetime density function. In information theory, Shannon entropy [10] plays a vital role in measuring the index of dispersion, volatility, or uncertainty associated with a random variable $X$. Shannon entropy for a nonnegative continuous random variable $X$ is given by

$$
H(X)=-E\left(\ln f_{X}(X)\right)=-\int_{0}^{\infty} f_{X}(x) \ln f_{X}(x) d x
$$

Ebrahimi [11] has defined the uncertainty of residual lifetime distributions $H(X ; t)$ by truncating the distributions below some point $t$ of a component as follows:

$$
\begin{aligned}
H(X ; t) & =-\int_{t}^{\infty} \frac{f_{X}(x)}{\bar{F}_{X}(t)} \ln \frac{f_{X}(x)}{\bar{F}_{X}(t)} d x \\
& =\ln \bar{F}_{X}(t)-\frac{1}{\bar{F}_{X}(t)} \int_{t}^{\infty} f_{X}(x) \ln f_{X}(x) d x \\
& =1-\frac{1}{\bar{F}_{X}(t)} \int_{t}^{\infty} f_{X}(x) \ln \lambda_{X}(x) d x,
\end{aligned}
$$

where $\lambda_{X}(x)$ is the failure rate or hazard rate defined by $f_{X}(x) / \bar{F}_{X}(x)$. Clearly for $t=0, H(X ; 0)$ represents the Shannon uncertainty contained in $X$.

Di Crescenzo and Longobardi [12] have introduced past entropy over $(0, t)$, since it is reasonable to presume that in many realistic situations uncertainty is not necessarily related to the future but can also refer to the past. They have also shown the necessity of past entropy and its relation with the residual entropy. If $X$ denotes the lifetime of an item or of a living organism, then past entropy (or uncertainty of lifetime distribution) of an item is defined as

$$
\begin{aligned}
\bar{H}(X ; t) & =-\int_{0}^{t} \frac{f_{X}(x)}{F_{X}(t)} \ln \frac{f_{X}(x)}{F_{X}(t)} d x \\
& =\ln F_{X}(t)-\frac{1}{F_{X}(t)} \int_{0}^{t} f_{X}(x) \ln f_{X}(x) d x \\
& =1-\frac{1}{F_{X}(t)} \int_{0}^{t} f_{X}(x) \ln \tau_{X}(x) d x,
\end{aligned}
$$

where $\tau_{X}(x)$ is the reversed hazard rate of $X$ given by $f_{X}(x) / F_{X}(x)$. In this paper, we will obtain and study the residual and past entropy of the Morgenstern family for concomitants of ordered random variables. We will also consider the characterization results based on the entropy function for concomitants of ordered random variables based on residual lifetime distribution and the past life distribution. The organization of the paper is as follows. In Section 2, we obtain the distribution function for concomitants of ordered random variables of the Morgenstern family. In Section 3, we obtain the residual and past entropy of our model and study the linear transformation and the upper bound for concomitants of ordered random variables. Some characterization results are presented in Section 4. Finally, some conclusions and comments are given in Section 5.

\section{Distribution Function for Concomitants of Ordered Random Variables}

Let $\left(X_{i}, Y_{i}\right), i=1,2, \ldots, n$, be $n$ pairs of independent continuous nonnegative random variables from some bivariate population with $\mathrm{df} F(x, y)$. From (1), the conditional distribution function of $Y$ given $X=x$ is given by

$$
\begin{aligned}
& F_{Y \mid X}(y \mid x)=f_{X}^{-1}(x) \frac{\partial F_{X, Y}(x, y)}{\partial x} \\
& \quad=F_{Y}(y)\left[1+\alpha\left(1-2 F_{X}(x)\right)\left(1-F_{Y}(y)\right)\right] .
\end{aligned}
$$

For the Morgenstern family with conditional distribution function given by (11), the $\mathrm{df}$ of the concomitant of case-I of gos $Y_{[r, n, m, k]}, 1 \leq r \leq n$, is given by

$$
\begin{array}{r}
G_{[r, n, m, k]}(y)=\int_{0}^{\infty} F_{Y \mid X}(y \mid x) f_{(r, n, m, k)}(x) d x \\
=F_{Y}(y)\left[1-\alpha C^{*}(r, n, m, k)\left(1-F_{Y}(y)\right)\right],
\end{array}
$$

where $f_{(r, n, m, k)}(x)$ is the pdf of case-I of gos $X_{[r, n, m, k]}$ (see Kamps [3]), the $\mathrm{df}$ of the concomitant of case-I of dgos $Y_{d[r, n, m, k]}, 1 \leq r \leq n$, is given by

$$
\begin{gathered}
G_{d[r, n, m, k]}(y)=\int_{0}^{\infty} F_{Y \mid X}(y \mid x) f_{d(r, n, m, k)}(x) d x \\
=F_{Y}(y)\left[1+\alpha C^{*}(r, n, m, k)\left(1-F_{Y}(y)\right)\right],
\end{gathered}
$$


where $f_{d(r, n, m, k)}(x)$ is the pdf of case-I of dgos $X_{d[r, n, m, k]}$ (see Burkschat et al. [6]), the df of the concomitant of case-II of gos $Y_{[r, n, \widetilde{m}, k]}, 1 \leq r \leq n$, is given by

$$
\begin{aligned}
G_{[r, n, \widetilde{m}, k]}(y) & =\int_{0}^{\infty} F_{Y \mid X}(y \mid x) f_{(r, n, \widetilde{m}, k)}(x) d x \\
& =F_{Y}(y)\left[1-\alpha D_{[r, n, \widetilde{m}, k]}\left(1-F_{Y}(y)\right)\right],
\end{aligned}
$$

where $f_{(r, n, \widetilde{m}, k)}(x)$ is the pdf of case-II of gos $X_{[r, n, \widetilde{m}, k]}$ (see Kamps and Cramer [4]), and the df of the concomitant of case-II of dgos $Y_{d[r, n, \widetilde{m}, k]}, 1 \leq r \leq n$, is given by

$$
\begin{aligned}
G_{d[r, n, \widetilde{m}, k]}(y) & =\int_{0}^{\infty} F_{Y \mid X}(y \mid x) f_{d(r, n, \widetilde{m}, k)}(x) d x \\
& =F_{Y}(y)\left[1+\alpha D_{[r, n, \widetilde{m}, k]}\left(1-F_{Y}(y)\right)\right],
\end{aligned}
$$

where $f_{d(r, n, \widetilde{m}, k)}(x)$ is the pdf of case-II of dgos $X_{d[r, n, \widetilde{m}, k]}$ (see Kamps and Cramer [4] and Burkschat et al. [6]).

Equations (4) to (7) and (12) to (15) can be combined as follows:

$$
\begin{aligned}
& g_{Y_{r}^{*}}(y)=f_{Y}(y)\left[1+\alpha M_{r}^{*}\left(2 F_{Y}(y)-1\right)\right], \\
& G_{Y_{r}^{*}}(y)=f_{Y}(y)\left[1+\alpha M_{r}^{*}\left(1-F_{Y}(y)\right)\right],
\end{aligned}
$$

where

$$
\begin{aligned}
& Y_{r}^{*}=\left[\begin{array}{ll}
Y_{[r, n, m, k]}, & \text { for case-I of gos } \\
Y_{d[r, n, m, k]}, & \text { for case-I of dgos } \\
Y_{[r, n, \widetilde{m}, k]}, & \text { for case-II of gos } \\
Y_{d[r, n, \widetilde{m}, k]}, & \text { for case-II of dgos, }
\end{array}\right. \\
& M_{r}^{*}=\left[\begin{array}{ll}
C^{*}(r, n, m, k), & \text { for case-I of gos } \\
-C^{*}(r, n, m, k), & \text { for case-I of dgos } \\
D_{[r, n, \widetilde{m}, k]}, & \text { for case-II of gos } \\
-D_{[r, n, \widetilde{m}, k]}, & \text { for case-II of dgos. }
\end{array}\right.
\end{aligned}
$$

In the following section we will use the last equations to obtain and study the residual and past entropy of concomitants for Morgenstern family based on the types of gos.

\section{Residual and Past Entropy}

Noting that $\bar{G}_{Y_{r}^{*}}(t)=1-G_{Y_{r}^{*}}(t)$ and $\partial G_{Y_{r}^{*}}(t) / \partial t=$ $g_{Y_{r}^{*}}(t)$, then, from (16), (17), and (9), the residual entropy for concomitants of ordered random variables of Morgenstern family is given by the following theorem.

Theorem 1. For any absolutely continuous random variable $Y_{r}^{*}$, which is the concomitant of $r$ th ordered random variable of Morgenstern family defined in (18). From (9), $Y_{r}^{*}$ has a residual entropy $H\left(Y_{r}^{*} ; t\right)$ iff

$$
\begin{aligned}
& H\left(Y_{r}^{*} ; t\right)=\ln \bar{G}_{Y_{r}^{*}}(t)-\frac{1}{\bar{G}_{Y_{r}^{*}}(t)}\left[\left(1-\alpha M_{r}^{*}\right)\right. \\
& \cdot\left[\bar{F}_{Y}(t)\left(\ln \bar{F}_{Y}(t)-H(Y ; t)\right)\right]+2 \alpha M_{r}^{*} \phi_{f}(y ; t) \\
& \left.\quad+K_{1}(r, t, \alpha, n, m, k)\right],
\end{aligned}
$$

where

$$
\begin{gathered}
K_{1}(r, t, \alpha, n, m, k)=\frac{1}{2 \alpha M_{r}^{*}}\left[\frac { - 1 } { 4 } \left[\left(1+\alpha M_{r}^{*}\right)^{2}\right.\right. \\
\left.-\left(1+\alpha M_{r}^{*}\left(2 F_{Y}(t)-1\right)\right)^{2}\right]+\frac{1}{2}\left[\left(1+\alpha M_{r}^{*}\right)^{2}\right. \\
\cdot \ln \left(1+\alpha M_{r}^{*}\right)-\left(1+\alpha M_{r}^{*}\left(2 F_{Y}(t)-1\right)\right)^{2} \\
\left.\left.\cdot \ln \left(1+\alpha M_{r}^{*}\left(2 F_{Y}(t)-1\right)\right)\right]\right], \\
\phi_{f}(y ; t)=\int_{t}^{\infty} F_{Y}(y) f_{Y}(y) \ln f_{Y}(y) d y, \\
1 \leq r \leq n, \alpha \neq 0,-1 \leq \alpha \leq 1 .
\end{gathered}
$$

From (16), (17), and (10), the past entropy for concomitants of ordered random variables of Morgenstern family is given by the following theorem.

Theorem 2. For any absolutely continuous random variable $Y_{r}^{*}$, which is the concomitant of $r$ th ordered random variable of Morgenstern family defined in (18). From (10), $Y_{r}^{*}$ has a past entropy $\bar{H}\left(Y_{r}^{*} ; t\right)$ iff

$$
\begin{aligned}
& \bar{H}\left(Y_{r}^{*} ; t\right)=\ln G_{Y_{r}^{*}}(t)-\frac{1}{G_{Y_{r}^{*}}(t)}\left[\left(1-\alpha M_{r}^{*}\right)\right. \\
& \quad \cdot\left[F_{Y}(t)\left(\ln F_{Y}(t)-\bar{H}(Y ; t)\right)\right]+2 \alpha M_{r}^{*} \bar{\phi}_{f}(y ; t) \\
& \left.\quad+K_{2}(r, t, \alpha, n, m, k)\right],
\end{aligned}
$$

where

$$
\begin{aligned}
& K_{2}(r, t, \alpha, n, m, k) \\
& =\frac{1}{2 \alpha M_{r}^{*}}\left[\frac { - 1 } { 4 } \left[\left(1+\alpha M_{r}^{*}\left(2 F_{Y}(t)-1\right)\right)^{2}\right.\right. \\
& \left.-\left(1-\alpha M_{r}^{*}\right)^{2}\right]+\frac{1}{2}\left[\left(1+\alpha M_{r}^{*}\left(2 F_{Y}(t)-1\right)\right)^{2}\right. \\
& \cdot \ln \left(1+\alpha M_{r}^{*}\left(2 F_{Y}(t)-1\right)\right)-\left(1-\alpha M_{r}^{*}\right)^{2} \\
& \left.\left.\cdot \ln \left(1-\alpha M_{r}^{*}\right)\right]\right], \\
& \bar{\phi}_{f}(y ; t)=\int_{0}^{t} F_{Y}(y) f_{Y}(y) \ln f_{Y}(y) d y, \\
& \quad 1 \leq r \leq n, \alpha \neq 0,-1 \leq \alpha \leq 1 .
\end{aligned}
$$

The following proposition gives the values of the functions $H\left(Y_{r}^{*} ; t\right)$ and $\bar{H}\left(Y_{r}^{*} ; t\right)$ under linear transformation.

Proposition 3. For any absolutely continuous random variable $Y_{r}^{*}$, which is the concomitant of $r$ th ordered random variable of Morgenstern family defined in (18), define $Z_{r}^{*}=$ $a Y_{r}^{*}+b$, where $a>0$ and $b \geq 0$ are constants. Then, for $t>b$, 
(1)

$$
\begin{aligned}
& H\left(Z_{r}^{*} ; t\right)=\ln \bar{G}_{Y_{r}^{*}}\left(\frac{t-b}{a}\right)-\frac{1}{\bar{G}_{Y_{r}^{*}}((t-b) / a)}[(1 \\
& \left.-\alpha M_{r}^{*}\right) \\
& \cdot\left[\bar{F}_{Y}\left(\frac{t-b}{a}\right)\left(\ln \bar{F}_{Y}\left(\frac{t-b}{a}\right)-H\left(Y ; \frac{t-b}{a}\right)\right)\right. \\
& \left.-\bar{F}_{Y}\left(\frac{t-b}{a}\right) \ln a\right]+2 \alpha M_{r}^{*}\left[\phi_{f}\left(y ; \frac{t-b}{a}\right)\right. \\
& \left.-\left(1-F_{Y}^{2}\left(\frac{t-b}{a}\right)\right) \frac{\ln a}{2}\right] \\
& \left.+K_{1}\left(r, \frac{t-b}{a}, \alpha, n, m, k\right)\right],
\end{aligned}
$$

$$
\begin{gathered}
\bar{H}\left(Z_{r}^{*} ; t\right)=\ln G_{Y_{r}^{*}}\left(\frac{t-b}{a}\right)-\frac{1}{G_{Y_{r}^{*}}((t-b) / a)}[(1 \\
\left.-\alpha M_{r}^{*}\right) \\
\cdot\left[F_{Y}\left(\frac{t-b}{a}\right)\left(\ln F_{Y}\left(\frac{t-b}{a}\right)-\bar{H}\left(Y ; \frac{t-b}{a}\right)\right)\right. \\
\left.-F_{Y}\left(\frac{t-b}{a}\right) \ln a\right]+2 \alpha M_{r}^{*}\left[\bar{\phi}_{f}\left(y ; \frac{t-b}{a}\right)\right. \\
\left.\left.-F_{Y}^{2}\left(\frac{t-b}{a}\right) \frac{\ln a}{2}\right]+K_{2}\left(r, \frac{t-b}{a}, \alpha, n, m, k\right)\right] .
\end{gathered}
$$

3.1. Upper Bound for Residual and Past Entropy. We derive the upper bound for the residual and past entropy under the condition that the pdf for $Y_{r}^{*}$, defined in (18), is less than 1 . Note that

$$
\begin{aligned}
H\left(Y_{r}^{*} ; t\right)= & -\int_{t}^{\infty} \frac{g_{Y_{r}^{*}}(y)}{\bar{G}_{Y_{r}^{*}}(t)} \ln \frac{g_{Y_{r}^{*}}(y)}{\bar{G}_{Y_{r}^{*}}(t)} d y \\
= & \ln \bar{G}_{Y_{r}^{*}}(t) \\
& -\frac{1}{\bar{G}_{Y_{r}^{*}}(t)} \int_{t}^{\infty} g_{Y_{r}^{*}}(y) \ln g_{Y_{r}^{*}}(y) d y .
\end{aligned}
$$

We know that, for $t>0, \ln \bar{G}_{Y_{r}^{*}}(t) \leq 0$. Using this we get

$$
\begin{aligned}
H\left(Y_{r}^{*} ; t\right) & \leq-\frac{1}{\bar{G}_{Y_{r}^{*}}(t)} \int_{t}^{\infty} g_{Y_{r}^{*}}(y) \ln g_{Y_{r}^{*}}(y) d y \\
& \leq-\frac{1}{\bar{G}_{Y_{r}^{*}}(t)} \int_{0}^{\infty} g_{Y_{r}^{*}}(y) \ln g_{Y_{r}^{*}}(y) d y .
\end{aligned}
$$

Hence,

$$
H\left(Y_{r}^{*} ; t\right) \leq \frac{H\left(Y_{r}^{*}\right)}{\bar{G}_{Y_{r}^{*}}(t)},
$$

with equality in which when $t \rightarrow 0, H\left(Y_{r}^{*}\right)$ is the Shannon entropy based on the concomitant of ordered random variables.

Next, we calculate an upper bound for the past entropy. We have

$$
\begin{aligned}
\bar{H}\left(Y_{r}^{*} ; t\right)= & -\int_{0}^{t} \frac{g_{Y_{r}^{*}}(y)}{G_{Y_{r}^{*}}(t)} \ln \frac{g_{Y_{r}^{*}}(y)}{G_{Y_{r}^{*}}(t)} d y \\
= & \ln G_{Y_{r}^{*}}(t) \\
& -\frac{1}{G_{Y_{r}^{*}}(t)} \int_{0}^{t} g_{Y_{r}^{*}}(y) \ln g_{Y_{r}^{*}}(y) d y .
\end{aligned}
$$

We know that, for $t>0, \ln G_{Y_{r}^{*}}(t) \leq 0$. Using this we get

$$
\begin{aligned}
\bar{H}\left(Y_{r}^{*} ; t\right) & \leq-\frac{1}{G_{Y_{r}^{*}}(t)} \int_{0}^{t} g_{Y_{r}^{*}}(y) \ln g_{Y_{r}^{*}}(y) d y \\
& \leq-\frac{1}{G_{Y_{r}^{*}}(t)} \int_{0}^{\infty} g_{Y_{r}^{*}}(y) \ln g_{Y_{r}^{*}}(y) d y .
\end{aligned}
$$

Hence,

$$
\bar{H}\left(Y_{r}^{*} ; t\right) \leq \frac{H\left(Y_{r}^{*}\right)}{G_{Y_{r}^{*}}(t)},
$$

with equality in which when $t \rightarrow 0, H\left(Y_{r}^{*}\right)$ is the Shannon entropy based on the concomitant of ordered random variables.

\section{Some Characterization Results}

Gupta et al. [13] have studied characterizations of residual and past entropy of order statistics. Here, in this section, we present some characterization results based on residual and past entropy of the concomitant of ordered random variables.

Consider a problem of finding sufficient condition for the uniqueness of the solution of the initial value problem (IVP):

$$
\begin{aligned}
\frac{d y}{d x} & =f(x, y), \\
y\left(x_{0}\right) & =y_{0},
\end{aligned}
$$

where $f$ is a given function of two variables whose domain is a region $D \subset \mathbb{R}^{2},\left(x_{0}, y_{0}\right)$ is a specified point in $D$, and $y$ is the unknown function. By the solution of the IVP on an interval $I \subset \mathbb{R}$, we mean a function $\phi(x)$ such that (i) $\phi$ is differentiable on $I$, (ii) the growth of $\phi$ lies in $D$, (iii) $\phi\left(x_{0}\right)=$ $y_{0}$, and (iv) $\phi^{\prime}(x)=f\left(x, \phi\left(x_{0}\right)\right)$, for all $x \in I$. The following theorem together with other results will help in proving our characterization result. 
Theorem 4. Let the function $f$ be defined and continuous in a domain $D \subset \mathbb{R}^{2}$, and let $f$ satisfy a Lipschitz condition (with respect to $y$ ) in D; namely,

$$
\left|f\left(x, y_{1}\right)-f\left(x, y_{2}\right)\right| \leq k\left|y_{1}-y_{2}\right|, \quad k>0,
$$

for every point $\left(x, y_{1}\right)$ and $\left(x, y_{2}\right)$ in $D$. Then the function $y=\phi(x)$ satisfies the initial value problem $y^{\prime}=f(x, y)$ and $\phi\left(x_{0}\right)=y_{0}, x \in I$, is unique.

Proof (see Gupta and Kirmani [14]). For any function $f(x, y)$ of two variables defined in $D \subset \mathbb{R}^{2}$, we now present a sufficient condition which guarantees that the Lipschitz condition is satisfied in $D$.

Lemma 5. Suppose that the function $f$ is continuous in a convex region $D \subset \mathbb{R}^{2}$. Suppose further that $\partial f / \partial y$ exists and is continuous in $D$. The function $f$ satisfies Lipschitz condition in $D$.

Proof (see Gupta and Kirmani [14]). We now present our two characterization results.

Theorem 6. Let $Y_{r}^{*}$ be a nonnegative continuous random variable, defined in (18), with distribution function $G_{Y_{r}^{*}}(\cdot)$ defined in (17). Let the residual entropy of the concomitant of rth ordered random variable be denoted by $H\left(Y_{r}^{*} ; t\right)<\infty$, $t \geq 0$. Then $H\left(Y_{r}^{*} ; t\right)$ characterizes the distribution.

Proof. Suppose there exist two functions $G_{Y_{r_{1}}^{*}}$ and $G_{Y_{r_{2}}^{*}}$ such that

$$
H\left(Y_{r_{1}}^{*} ; t\right)=H\left(Y_{r_{2}}^{*} ; t\right)
$$

for all $t>0$. Then

$$
\begin{aligned}
H^{\prime}\left(Y_{r_{i}}^{*} ; t\right)=\lambda_{Y_{r_{i}}^{*}}(t)\left[H\left(Y_{r_{i}}^{*} ; t\right)-1+\lambda_{Y_{r_{i}}^{*}}(t)\right] & \\
& i=1,2,
\end{aligned}
$$

where $\lambda_{Y_{r_{i}}^{*}}(t)=g_{Y_{r_{i}}^{*}}(t) / \bar{G}_{Y_{r_{i}}^{*}}(t)$ is the hazard rate of the concomitant of $r$ th ordered random variable, for $i=1,2$. Differentiating the above equation with respect to $t$ and simplifying, we get

$$
\begin{gathered}
\lambda_{Y_{r_{i}}^{*}}^{\prime}(t)=\frac{\lambda_{Y_{r_{i}}^{*}}(t)}{\lambda_{Y_{r_{i}}^{*}}(t)+H^{\prime}\left(Y_{r_{i}}^{*} ; t\right)}\left[H^{\prime \prime}\left(Y_{r_{i}}^{*} ; t\right)\right. \\
\left.-\lambda_{Y_{r_{i}}^{*}}(t) H^{\prime}\left(Y_{r_{i}}^{*} ; t\right)\right], \quad i=1,2 .
\end{gathered}
$$

Suppose now that

$$
H\left(Y_{r_{1}}^{*} ; t\right)=H\left(Y_{r_{2}}^{*} ; t\right)=g(t)
$$

Then, for all $t \geq 0$,

$$
\lambda_{Y_{r_{i}}^{*}}^{\prime}(t)=\psi\left(t, \lambda_{Y_{r_{i}}^{*}}(t)\right)
$$

for $i=1,2$, where

$$
\psi(t, y)=\frac{y}{y+g^{\prime}(t)}\left[g^{\prime \prime}(t)-y g^{\prime}(t)\right]
$$

It follows from Theorem 4 and Lemma 5 that $\lambda_{Y_{r_{1}}^{*}}^{\prime}(t)=$ $\lambda_{Y_{r_{2}}^{*}}^{\prime}(t)$, which prove the characterization result.

Theorem 7. Let $Y_{r}^{*}$ be a nonnegative continuous random variable, defined in (18), with distribution function $G_{Y_{r}^{*}}(\cdot)$ defined in (17). Let the past entropy of the concomitant of $r$ th ordered random variable be denoted by $\bar{H}\left(Y_{r}^{*} ; t\right)<\infty, t \geq 0$. Then $\bar{H}\left(Y_{r}^{*} ; t\right)$ characterizes the distribution.

Proof. By the same way of the last theorem, where $\tau_{Y_{r_{i}^{*}}^{*}}(t)=$ $g_{Y_{r_{i}}^{*}}(t) / G_{Y_{r_{i}}^{*}}(t)$ is the reversed hazard rate of the concomitant of $r$ th ordered random variable, for $i=1,2$.

Next we present a characterization result of the linear mean residual family of the concomitant of $r$ th ordered random variable based on Theorem 6 . The linear mean residual life of the concomitant of $r$ th ordered random variable is given by $\mu_{Y_{r}}(t)=a+b t, a>0, b>-1$. It can be verified that the corresponding failure rate is given by $\lambda_{Y_{r}^{*}}(t)=(1+b) /(a+$ $b t)$. The linear mean residual family of a distribution has been studied among others by Hall and Wellner [15], Oakes and Dasu [16], and Gupta and Kirmani [14]. It includes the exponential distribution for $b=0$ and the power distribution for $-1<b<0$. Gupta et al. [13] have studied the linear mean residual life of a distribution based on order statistics.

Theorem 8. Let $Y_{r}^{*}$ be a nonnegative continuous random variable, defined in (18). Then $H\left(Y_{r}^{*} ; t\right)=1+b /(1+b)-$ $\ln \lambda_{Y_{r}^{*}}(t)$ if and only if $\lambda_{Y_{r}^{*}}(t)=(1+b) /(a+b t)$.

Proof. From (9), we have

$$
\begin{aligned}
H\left(Y_{r}^{*} ; t\right)= & 1-\frac{1}{\bar{G}_{Y_{r}^{*}}(t)} \int_{t}^{\infty} g_{Y_{r}^{*}}(y) \ln \lambda_{Y_{r}^{*}}(y) d y \\
= & 1-\ln (1+b) \\
& +\frac{1}{\bar{G}_{Y_{r}^{*}}(t)} \int_{t}^{\infty} g_{Y_{r}^{*}}(y) \ln (a+b y) d y .
\end{aligned}
$$

To find $\int_{t}^{\infty} g_{Y_{r}^{*}}(y) \ln (a+b y) d y$, first, we want to find

$$
\begin{aligned}
T(l, t) & =\int_{t}^{\infty} g_{Y_{r}^{*}}(y)(a+b y)^{l} d y \\
& =\int_{t}^{\infty} \bar{G}_{Y_{r}^{*}}(y)\left(\frac{1+b}{a+b y}\right)(a+b y)^{l} d y \\
& =\frac{1+b}{b+1-b l}(a+b t)^{l} \bar{G}_{Y_{r}^{*}}(t) .
\end{aligned}
$$


Taking the derivative of the above equation with respect to $l$ and evaluating at $l=0$, we get $\int_{t}^{\infty} g_{Y_{r}^{*}}(y) \ln (a+b y) d y=$ $(b /(1+b)+\ln (a+b t)) \bar{G}_{Y_{r}^{*}}(t)$. Substituting the pervious results into (40) we get

$$
\begin{aligned}
H\left(Y_{r}^{*} ; t\right) & =1+\frac{b}{1+b}-\ln \frac{1+b}{a+b t} \\
& =1+\frac{b}{1+b}-\ln \lambda_{Y_{r}^{*}}(t) .
\end{aligned}
$$

To prove the converse, (35) gives

$$
\begin{aligned}
& H^{\prime}\left(Y_{r}^{*} ; t\right)=\lambda_{Y_{r}^{*}}(t)\left[H\left(Y_{r}^{*} ; t\right)-1+\lambda_{Y_{r}^{*}}(t)\right] \\
& \quad=\lambda_{Y_{r}^{*}}(t)\left[1+\frac{b}{1+b}-\ln \lambda_{Y_{r}^{*}}(t)-1+\lambda_{Y_{r}^{*}}(t)\right] \\
& \quad=\lambda_{Y_{r}^{*}}(t)\left[\frac{b}{1+b}\right] .
\end{aligned}
$$

Since $H^{\prime}\left(Y_{r}^{*} ; t\right)=-\lambda_{Y_{r}^{*}}^{\prime}(t) / \lambda_{Y_{r}^{*}}(t)$, then we get

$$
\lambda_{Y_{r}^{*}}^{\prime}(t)+\frac{b}{1+b} \lambda_{Y_{r}^{*}}^{2}(t)=0
$$

whose solution is

$$
\lambda_{Y_{r}^{*}}(t)=\frac{1+b}{a+b t} .
$$

\section{Conclusion and Comments}

The different types of gos are considered in one model based on the concomitants for Morgenstern family. From this model, we derived an analytical expression of the residual and past lifetime distribution and find some formulas of it. The dynamic entropy measures based on the concomitant of ordered random variables characterize uniquely the underlying distribution and are also bounded above in terms of Shannon entropy of the concomitant of the different types of gos. Stochastic comparisons can also be studied easily for such model.

\section{Conflict of Interests}

The authors declare that they have no conflict of interests.

\section{References}

[1] N. L. Johnson and S. Kotz, "On some generalized FarlieGumbel-Morgenstern distributions," Communications in Statistics, vol. 4, no. 5, pp. 415-427, 1975.

[2] H. A. David, M. J. O'Connell, and S. S. Yang, "Distribution and expected value of the rank of a concomitant of an order statistic," The Annals of Statistics, vol. 5, no. 1, pp. 216-223, 1977.

[3] U. Kamps, "A concept of generalized order statistics," Journal of Statistical Planning and Inference, vol. 48, no. 1, pp. 1-23, 1995.
[4] U. Kamps and E. Cramer, "On distributions of generalized order statistics," Statistics, vol. 35, no. 3, pp. 269-280, 2001.

[5] P. Pawlas and D. Szynal, "Recurrence relations for single and product moments of lower generalized order statistics from the inverse Weibull distribution," Demonstratio Mathematica, vol. 34, no. 2, pp. 353-358, 2001.

[6] M. Burkschat, E. Cramer, and U. Kamps, "Dual generalized order statistics," Metron, vol. 61, no. 1, pp. 13-26, 2003.

[7] M. I. Beg and M. Ahsanullah, "Concomitants of generalized order statistics from Farlie-Gumbel-Morgenstern distributions," Statistical Methodology, vol. 5, no. 1, pp. 1-20, 2008.

[8] Nayabuddin, "Concomitants of dual generalized order statistics from Farlie Gumbel Morgenstern type bivariate power function distribution," Journal of Global Research in Mathematical Archives, vol. 1, no. 8, pp. 79-90, 2013.

[9] M. M. Mohie El-Din, M. M. Ameina, and M. S. Mohamed, "Concomitants of case-II of generalized order statistics from Farlie-Gumbel-Morgenstern distributions," Journal of Statistics Applications and Probability, vol. 3, no. 3, pp. 345-353, 2015.

[10] C. E. Shannon, "A mathematical theory of communication," The Bell System Technical Journal, vol. 27, no. 3, pp. 379-423, 1948.

[11] N. Ebrahimi, "How to measure uncertainty in the residual life time distribution," Sankhyā. The Indian Journal of Statistics A, vol. 58, no. 1, pp. 48-56, 1996.

[12] A. Di Crescenzo and M. Longobardi, "Entropy-based measure of uncertainty in past lifetime distributions," Journal of Applied Probability, vol. 39, no. 2, pp. 434-440, 2002.

[13] R. C. Gupta, H. C. Taneja, and R. Thapliyal, "Stochastic comparisons of residual entropy of order statistics and some characterization results," Journal of Statistical Theory and Applications, vol. 13, no. 1, pp. 27-37, 2014.

[14] R. C. Gupta and S. N. U. A. Kirmani, "Characterization based on convex conditional mean function," Journal of Statistical Planning and Inference, vol. 138, no. 4, pp. 964-970, 2008.

[15] W. J. Hall and J. A. Wellner, "Mean residual life," in Statistics and Related Topics, pp. 169-184, North-Holland, Amsterdam, The Netherlands, 1981.

[16] D. Oakes and T. Dasu, "A note on residual life," Biometrika, vol. 77, no. 2, pp. 409-410, 1990. 


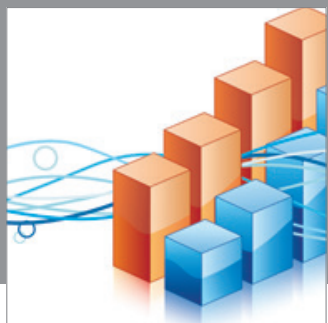

Advances in

Operations Research

mansans

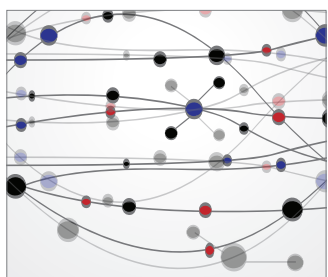

The Scientific World Journal
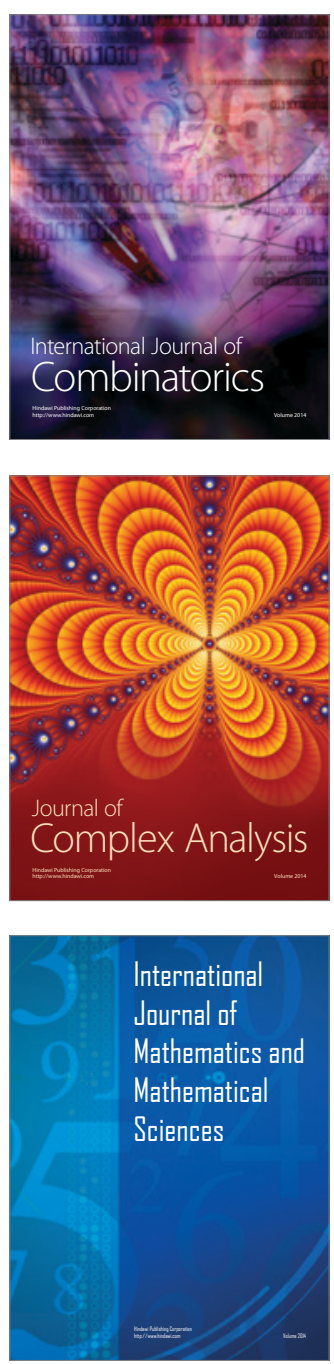
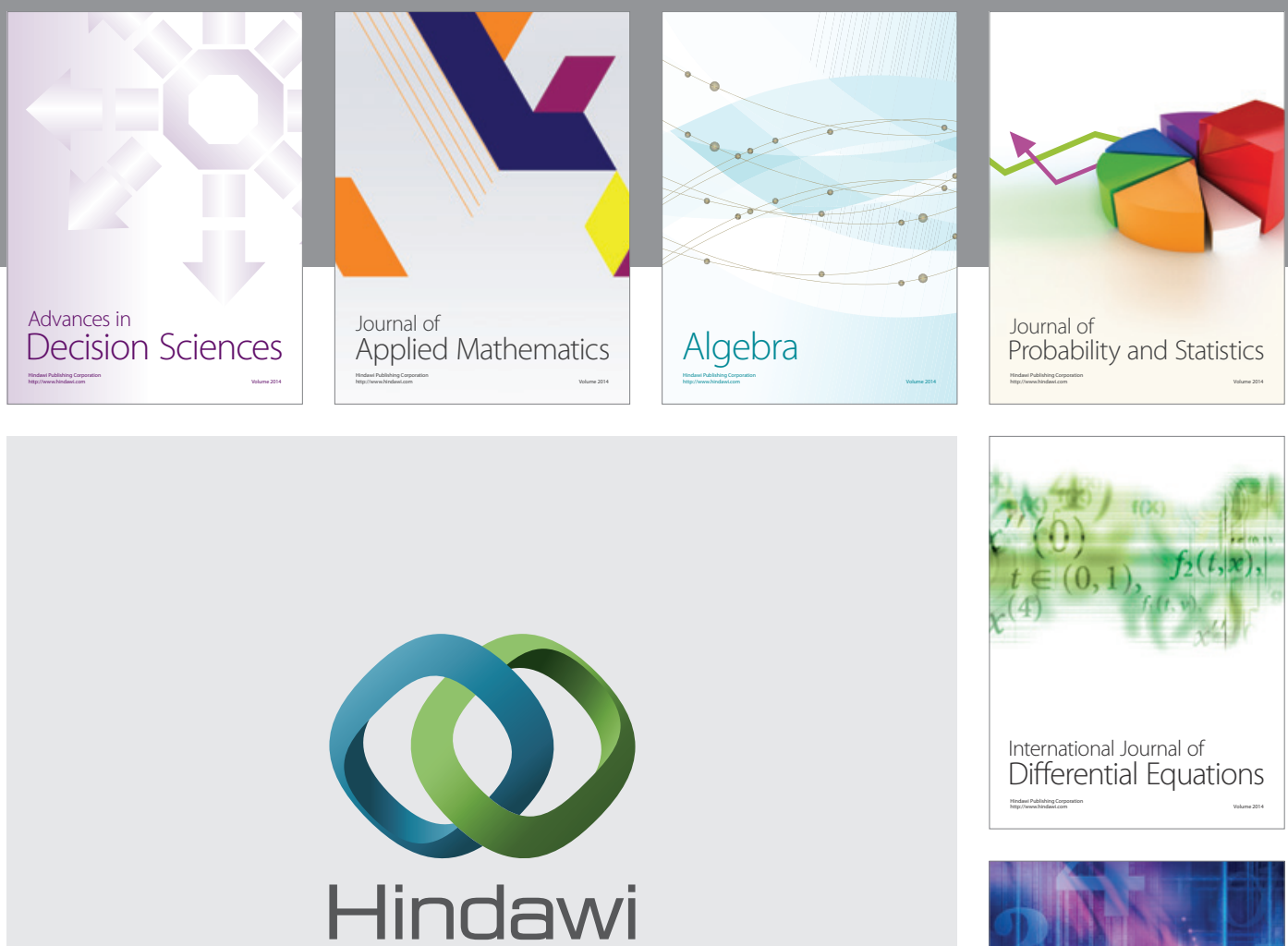

Submit your manuscripts at http://www.hindawi.com
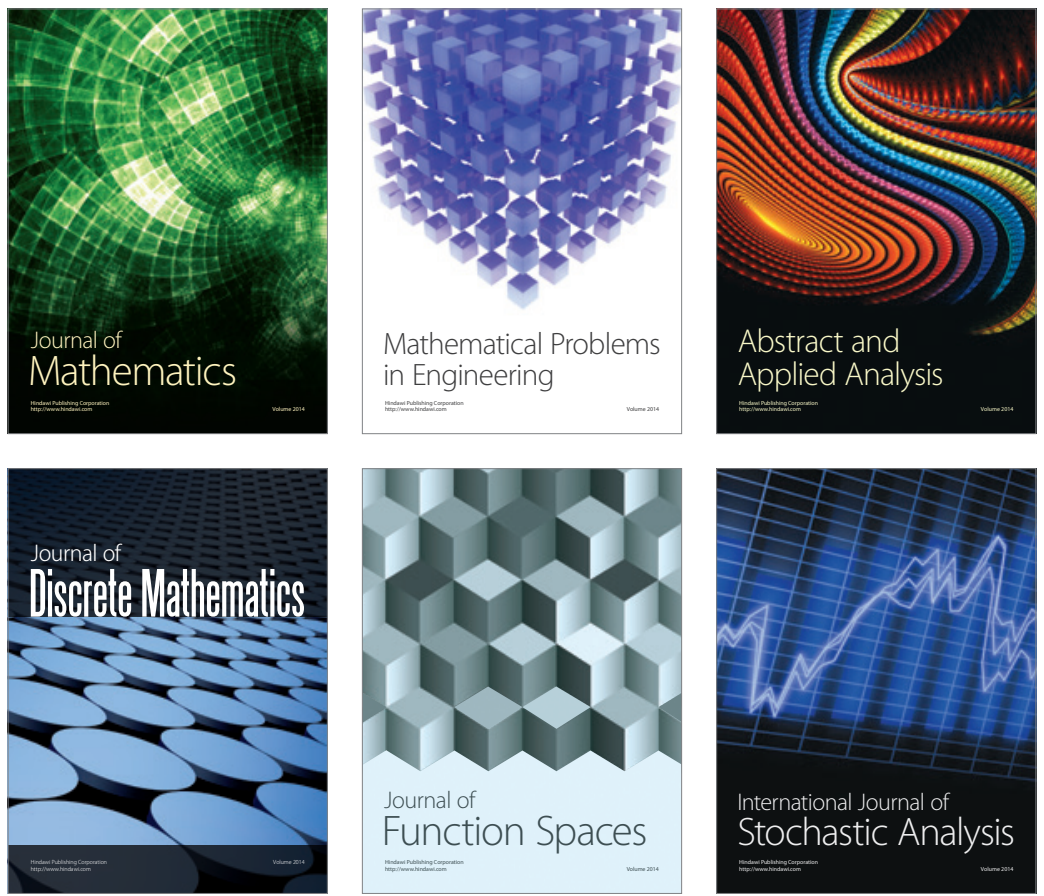

Journal of

Function Spaces

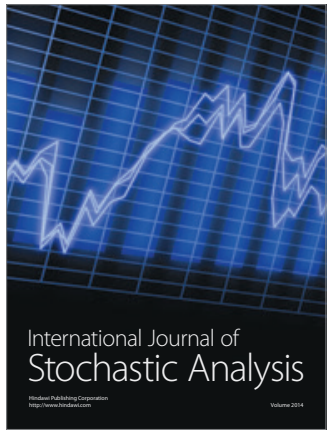

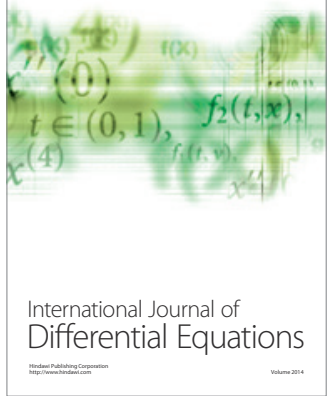
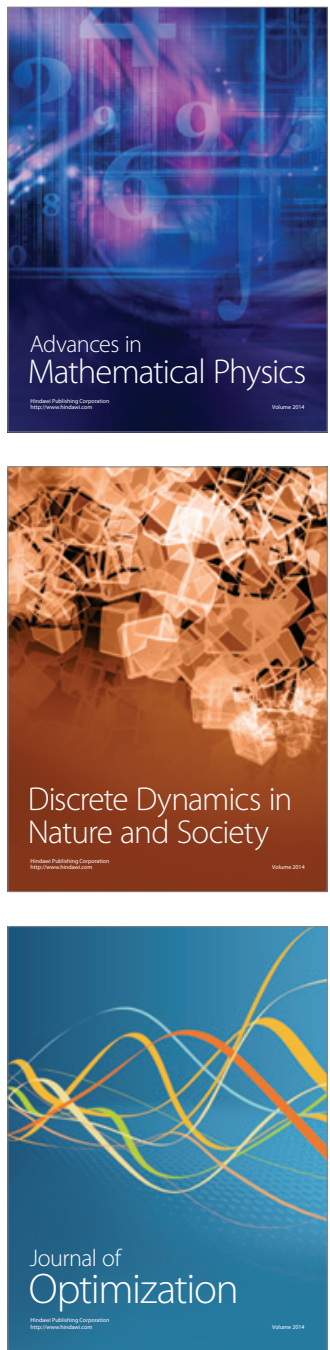